\title{
Cognitive-Behavioral Group Therapy for Youths with Anxiety Disorders in the Community: Effectiveness in Low and Middle Income Countries
}

\author{
Maria Augusta Mansur de Souza, Giovanni A. Salum, Rafaela Behs Jarros, \\ Luciano Isolan, Roberta Davis, Daniela Knijnik, Gisele Gus Manfro and Elizeth Heldt
}

Federal University of Rio Grande do Sul (UFRGS), Brazil

\begin{abstract}
Background: Although cognitive-behavioral therapy (CBT) is established as a first line treatment for anxiety disorders in children and adolescents, there is little evidence about the effectiveness of CBT protocols in cases identified in the community in low and middle income countries (LaMICs). Aims: To evaluate the effectiveness of group CBT protocol for youths with anxiety disorders identified in a community sample in LaMICs. Method: A total of 14 sessions of group CBT for youths and 2 concurrent sessions for parents based on Kendall's Coping Cat program were offered. Participants were selected from a crosssectional community study; 45 subjects fulfilled inclusion criteria and 28 agreed to participate in the open clinical trial. Treatment effectiveness was evaluated with standard clinical, selfand parent-rated measures of anxiety, depression, externalizing symptoms and quality of life (QoL). Results: Twenty youths completed the protocol. All scales showed an improvement of anxiety and reduction in externalizing symptoms over time, with a moderate to large effect size $(d=0.59$ to $2.06 ; p<.05)$, but not in depressive symptoms or QoL. Conclusions: Consistent with previous evidence, group CBT is effective in treating anxiety disorders in youths. Results encourage further randomized clinical trials using CBT protocols adapted and developed to be used in LaMICs.
\end{abstract}

Keywords: Cognitive-behavioral therapy, anxiety disorders, youth, group therapy.

\section{Introduction}

Anxiety disorders are among the most common psychiatric disorders in children and adolescents (Beesdo, Knappe and Pine, 2009; Merikangas et al., 2010). Epidemiological studies describe prevalence rates ranging from 2 to $4 \%$ for current or 3-month pediatric anxiety disorders, and 6 to 12-month prevalence rates vary between 10 and 20\% (Costello, Egger and Angold, 2005). In addition to significant negative impact on social and emotional development of children (Kessler et al., 2005), anxiety disorders tend to follow a chronic and fluctuating course in adulthood (Merikangas et al., 2010). Therefore early identification and effective treatment can reduce the impact of disease on social and academic functioning

Reprint requests to Maria Mansur de Souza, UFRGS, Lucas de Oliveira, 2776/202 Porto Alegre, Rio Grande do Sul 90460000, Brazil. E-mail: ma.mansur@terra.com.br 
(Kendall, Khanna, Edson, Cummings and Harris, 2011; Klein, 2009) and on development of psychiatric disorders in adulthood (Connolly, Bernstein and Issues, 2007).

Anxiety disorder treatment has been the focus of several studies with important advances in the last 20 years (March, 2011). Selective Serotonin Reuptake Inhibitors (SSRIs) and Cognitive-Behavioral Therapy (CBT) are both considered first line treatments for anxiety disorders (Rynn et al., 2011; Walkup et al., 2008). A recent review of controlled trials showed a remission rate of around $56 \%$ for CBT using conservative intention-to-treat criteria versus $28.2 \%$ for controls (James, Soler and Weatherall, 2007). However, the majority of studies were conducted in specific research centers in high income countries (Connolly et al., 2007).

Although first line options are easily accessible around the world, in low and middle income countries (LaMICs) such as Brazil, CBT is usually limited to research centers and specialized care. We believe that the small number of qualified therapists and the lack of CBT protocols designed for these specific populations may contribute to its underutilization. Dissemination of effective techniques is an important new research field and adequate protocols are a starting point for this (Kendall et al., 2011; McLean and Foa, 2011).

CBT protocols have been developed in Brazil and shown to be effective with adults with anxiety disorders in the short (Heldt et al., 2003; Cordioli et al., 2003) and longer term (Braga, Manfro, Niederauer and Cordioli, 2010; Heldt et al., 2011). However, CBT programmes for youth with anxiety disorders have not been tested in Brazil and there is a notable lack of qualified health professionals capable of conducting CBT with children and adolescents

The CBT group programme used in this study was based on a recognized CBT protocol for anxious children: the Coping Cat program (Kendall and Hedtke, 2006a,b). This study is not a cross-cultural adaptation of a manual, but rather it uses previously validated CBT techniques to develop a feasible protocol adapted to Brazilian socioeconomic and cultural reality, focusing on anxiety, with evidence of similar responses to treatment for separation anxiety disorder (SeAD), social anxiety disorder (SoAD), panic disorder (PD), and generalized anxiety disorder (GAD) in youths. Our objective is to evaluate the effectiveness of group CBT protocol for youths with anxiety disorders identified in a community sample in LaMICs.

\section{Method}

This is an open clinical trial designed to evaluate the effectiveness of group CBT for anxiety disorders in youth and was undertaken from 2009 to 2010. The study was approved by the Research and Ethics Committee of Hospital de Clínicas de Porto Alegre ( $\left.{ }^{\circ} 08-450\right)$. Primary caretakers and youths signed written informed consents to participate.

\section{Participants}

This study is part of a community cross-section study that screened a total of 2457 subjects from 10 to 17-years-old for anxiety disorders in the community with the Screen for Child and Adolescents Related Emotional Disorders (Isolan, Salum, Osowski, Amaro and Manfro, 2011). A sub-sample of 242 subjects were further evaluated with the Schedule for Affective Disorders and Schizophrenia for School-Age Children-Present and Lifetime Version (K-SADS-PL) (Kaufman et al., 1997), performed by independent child and adolescent psychiatrists or psychiatric residents receiving supervision (Salum et al., 2011). From those a total of $138(5.6 \%)$ were diagnosed with at least one anxiety disorder. 


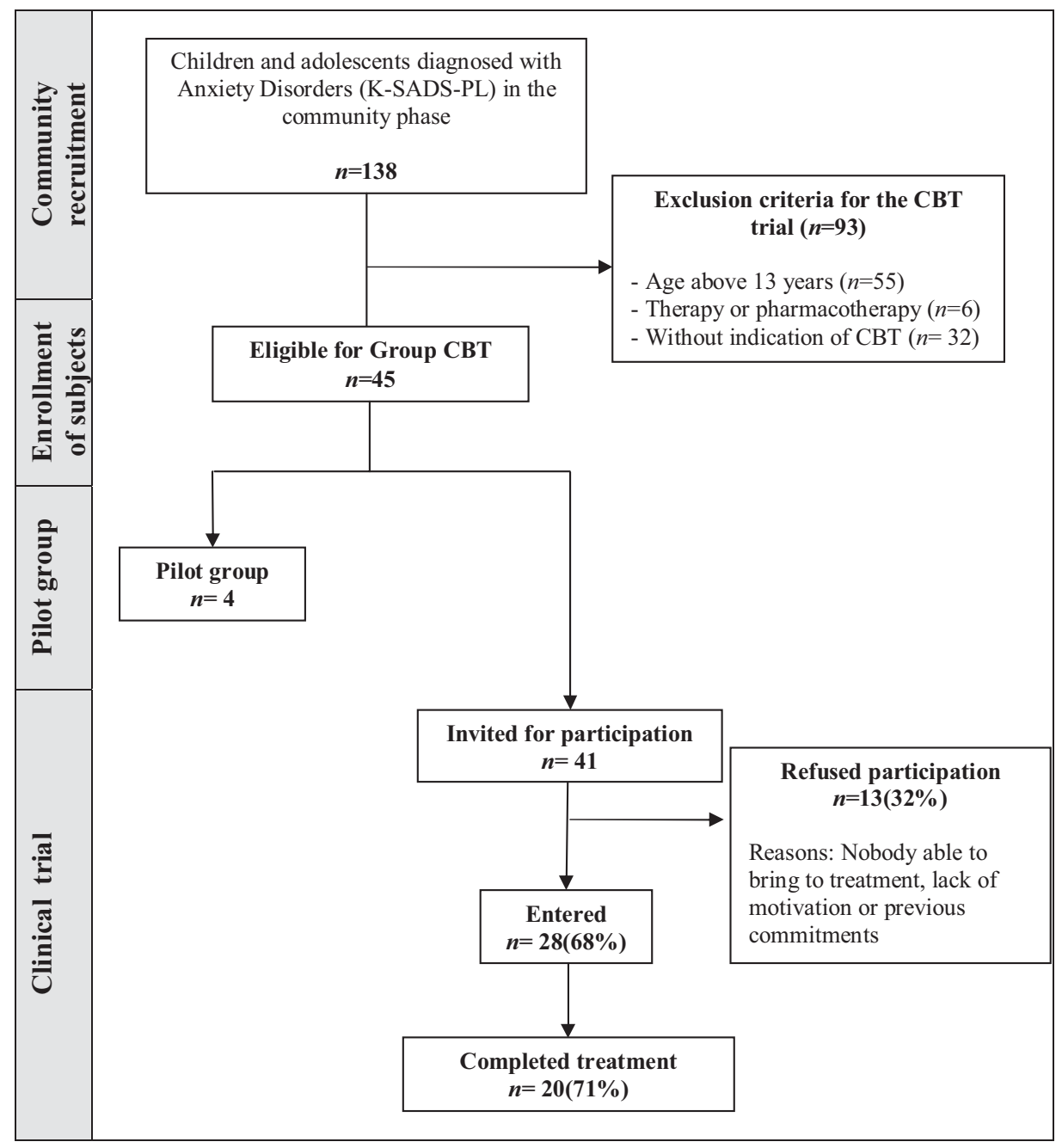

Figure 1. Flow chart of the study

In order to participate in the CBT trial, youths had to be between 10 to 13 years old, not receiving psychotherapy or psychopharmacological treatment at the time of recruitment, and be available to participate in group CBT for 14 weeks. The exclusion criteria include: severe comorbidities that demanded immediate treatment, main diagnosis of PTSD, OCD, psychotic disorders or pervasive developmental disorder, and mental retardation or cognitive dysfunction.

Forty-five subjects met inclusion criteria; 4 participated in a pilot group, with the remaining 41 being invited to participate in the main trial. Of these, 28 consented to take part in the research (see Figure 1). 


\section{Intervention}

The intervention was based on a recognized CBT protocol for anxious children, the Coping Cat program - Cognitive-Behavioral Therapy for Anxious Children: therapist manual for group treatment (Kendall and Hedtke, 2006a), and The Coping Cat Workbook (Kendall and Hedtke, 2006b).

The intervention consisted of 14 weekly 90 minute sessions. Two more concurrent sessions with parents, one in the seventh and another at the last session of treatment, were included. CBT was conducted by two clinical psychologists, supervised by researchers with more than 10 years of experience in group CBT. Before starting the open trial we undertook a pilot group $(n=4)$ to assess suitability for a Brazilian population and the time required to deliver the programme.

The group intervention aimed to: increase recognition and analysis of distorted thinking patterns that contribute to anxiety symptoms; develop coping strategies to deal with anxietyprovoking situations; recognize signs of anxiety as a signal to use the coping strategies and to decrease or eliminate avoidance that maintains anxiety (Kendall et al., 1997; Kendall and Hedtke, 2006a,b).

\section{Study procedures}

After the pilot CBT group was completed, 41 youths were invited to participate in the trial and were assessed by child and adolescent psychiatrists and through self-report questionnaires described below. These evaluations were conducted at three different time points: before starting treatment (baseline), in the middle (session 7), and at the end of treatment (session 14).

\section{Primary outcomes}

The Clinical Global Impression (CGI) rating scale was used as the primary dichotomous outcome. CGI is a clinician rating scale of severity that ranges from 1 (normal) to 7 (extremely ill), with lower scores indicating greater improvement, as compared with baseline (Guy, 1976). Remission rates were defined by a CGI $\leqslant 2$ in the previous month.

The Pediatric Anxiety Rating Scale (PARS) was used as the primary continuous outcome. With this scale, the clinician uses a checklist of questions regarding the symptoms of anxiety during the past week to elicit responses from parents and youths aged between 6-17 years. After completing this checklist, the clinician rates five general items: the severity of the child's distress due to anxiety, the frequency of anxiety, the degree to which the child avoided anxietyprovoking situations, and the degree to which the anxiety limited the child's participation in typical daily activities both at home and in other environments, such as school. Total scores on this scale range from 0 to 30 , with scores above 13 indicating clinically meaningful anxiety. Symptoms were confirmed through integration of both youth and parent information, rated by the evaluator (The Research Units on Pediatric Psychopharmacology Anxiety Study Group, 2002).

\section{Secondary outcomes}

The Screen for Child Anxiety Related Emotional Disorders (SCARED; Birmaher et al., 1997) is a child and parent self-report measure with 41 items that assess DSM-IV symptoms of 
SeAD, GAD, SoAD, PD, and school phobia. The two versions are for the child (SCAREDC) and parents (SCARED-P) respectively. The total score and each of the five factors of the SCARED Brazilian-Portuguese version showed good internal consistency, test-retest and construct validity (Isolan et al., 2011).

The Children's Global Assessment Scale (CGAS) provides a global impairment measure and functioning over the previous month. The scale ranges from 1 (lowest) to 100 (highest) and scores of 60 or lower are considered to indicate a need for treatment (Shaffer et al., 1983).

The Children's Depression Inventory (CDI) is a 27-item self-report measure assessing cognitive and somatic symptoms associated with depression in people aged from 7 to 17 years old (Golfeto and D'Oliveira, 2004). Items are rated on a 3-point scale, ranging from 0 to 2 , reflecting the degree of particular depressive symptoms over the past 2 weeks. With the sum of the scores of all items, we obtained a total score from 0 to 54 .

Youth Quality of Life Instrument-Research Version (YQOL-R) is a self-report questionnaire of 49 perceptual items used to perform a multidimensional assessment of the generic quality of life (QoL) (Edwards, Huebner, Connell and Patrick, 2002; Patrick, Edwards and Topolski, 2002). A conceptual model was used to organize QoL items for each domain and their associated facets, in an 11-point scale with anchors of 0 (Not at all) and 10 (A great deal or completely). The YQOL-R is considered a youth-centered questionnaire, focuses on positive aspects of health and perceptions, and allows cross-cultural comparisons.

The SNAP-IV was the version used in a Multimodality Treatment Study, which includes 26 items corresponding to Attention Deficit Disorder Hyperactivity (ADHD), and symptoms of Oppositional Defiant Disorder (ODD). The 4-point response is scored from 0-3, subscale scores for the ADHD and ODD are calculated by summing the scores on the items in the specific subset (e.g. Inattention) and dividing by the number of items in the subset (SerraPinheiro, Mattos and Angélica, 2008).

\section{Statistical analysis}

Data were described as count and percent $(\%)$, mean and standard deviations (SD). A Generalized Estimating Equations (GEE) model was performed taking into consideration the individual and therapy group levels testing time effect in the repeated measures at baseline, mid-point and endpoint evaluations. An unstructured correlation matrix was used as a robust estimator. Sequential Bonferroni adjustment for multiple comparisons was used to compare time points. All comparisons were adjusted for age and gender. These tests were complemented by presentation of within-group effect sizes (Cohen's $d=M_{1}-M_{2} / \sqrt{ } S D_{1}^{2}+$ $S D_{2}^{2}-2 \times r \times S D_{1} \times S D_{2}$ ). Tests were two-tailed, with a significance level of .05 and performed with SPSS 17.0.

\section{Results}

A total of $28(68 \%)$ patients were included in one of the four groups, with demographic characteristics and diagnosis being summarized in Table 1 . Twenty patients $(71 \%)$ completed the intervention with $9(45 \%)$ meeting criteria for remission (CGI $\leqslant 2)$. All clinician, self-rated and parent-rated scales showed an improvement over time, with a moderate to large effect (Cohen's d ranging from $=0.59$ to 2.09). In addition, there was a significant 
Table 1. Descriptive sample characteristics $(n=28)$

\begin{tabular}{lcc}
\hline Age (years) & 12 & $S D=0,77$ \\
Gender (female) & 22 & $79 \%$ \\
Anxiety disorders: & 3 & $11 \%$ \\
$\quad$ PD & 13 & $46 \%$ \\
SeAD & 11 & $39 \%$ \\
SoAD & 23 & $82 \%$ \\
$\quad$ GAD & & \\
Present comorbidities: & & \\
$\quad$ ADHD & 8 & $29 \%$ \\
$\quad$ ODD & 4 & $14 \%$ \\
Past comorbidities: & & \\
$\quad$ Enuresis & 4 & $14 \%$ \\
Depression & 6 & $21 \%$ \\
Anxiety disorders comorbidities: & & \\
1 & 6 & $21 \%$ \\
2 & 12 & $43 \%$ \\
3 & 9 & $32 \%$ \\
4 & 1 & $4 \%$ \\
\hline
\end{tabular}

Notes: Data are presented as $n(\%)$ and mean and Standard Deviation $(S D)$

Abbreviations: $\mathrm{ADHD}=$ Attention Deficit/Hyperactivity Disorder; $\mathrm{ODD}=$ Oppositional Defiant Disorder; PD $=$ Panic Disorder; SeAD = Separation Anxiety Disorder; SoAD = Social Anxiety Disorder; GAD $=$ Generalized Anxiety Disorders.

${ }^{*}$ Relative frequency superior to $10 \%$

improvement in symptoms of externalizing disorders, but not in depressive symptoms over time. No significant change was found in QoL scores (see Table 2).

\section{Discussion}

This study was a first attempt to clinically test a CBT protocol for anxiety disorders in Brazilian youth (Kendall et al., 1997; Kendall and Hedtke, 2006a,b). Our findings suggest that group CBT is an effective intervention for youth with anxiety disorders in a LaMIC.

Interestingly, CBT produced substantial treatment effects for anxiety symptoms measured by PARS and SCARED, although it did not result in a significant decrease in depressive symptoms nor an improvement in quality of life. There is evidence that depression is frequently a consequence of high levels of anxiety and something that starts in childhood (Merikangas et al., 2010; Roza, Hofstra, Van Der Ende and Verhulst, 2003). The mean age of our sample is around 12 years old and the mean of depressive symptoms was low, as verified by CDI, suggesting that perhaps we did not have enough power to show mild differences in depression scores (Kendall, Safford, Flannery-Schroeder and Webb, 2004).

Another interesting result was the significant decrease in externalizing symptoms measured by the SNAP-IV after the treatment. With regard to this finding, around $30 \%$ of the sample 
Table 2. Estimated marginal means of each outcome over the course of treatment

\begin{tabular}{|c|c|c|c|c|c|c|c|c|c|c|c|}
\hline & \multicolumn{3}{|c|}{ Baseline } & \multicolumn{3}{|c|}{ Week 7} & \multicolumn{3}{|c|}{ Week 14} & \multirow{2}{*}{$\begin{array}{l}\text { GEE time } \\
\text { effect }\end{array}$} & \multirow{2}{*}{$\begin{array}{l}\text { Effec } \\
\text { Size* }^{*}\end{array}$} \\
\hline & EMM & CI95\% & & EMM & CI95\% & & EMM & CI95\% & & & \\
\hline \multicolumn{12}{|l|}{ Clinician rated: } \\
\hline PARS & $13.8^{\mathrm{a}}$ & 12.7 & 14.9 & $10.8^{b}$ & 9.6 & 11.9 & $7.2^{\mathrm{c}}$ & 5.6 & 8.7 & $<0.001$ & 1.57 \\
\hline CGI & $4.1^{\mathrm{a}}$ & 3.8 & 4.4 & $3.5^{\mathrm{b}}$ & 3.2 & 3.9 & $2.7^{\mathrm{c}}$ & 2.2 & 3.1 & $<0.001$ & 2.06 \\
\hline CGAS & $55.9^{\mathrm{a}}$ & 52.9 & 58.9 & $62.2^{\mathrm{b}}$ & 59.2 & 65.2 & $69.8^{c}$ & 65.3 & 74.4 & $<0.001$ & 1.93 \\
\hline \multicolumn{12}{|l|}{ Self-rated: } \\
\hline SCARED-C & $28.2^{\mathrm{a}}$ & 23.8 & 32.6 & $25.0^{\mathrm{a}, \mathrm{b}}$ & 21.3 & 28.8 & $21.6^{\mathrm{b}}$ & 18.2 & 25.1 & 0.012 & 0.66 \\
\hline \multicolumn{12}{|l|}{ Parent-rated: } \\
\hline SCARED-P & $29.2^{\mathrm{a}}$ & 24.4 & 34.1 & $28.2^{\mathrm{a}, \mathrm{b}}$ & 23.1 & 33.4 & $23.0^{\mathrm{b}}$ & 18.1 & 27.9 & $<0.001$ & 0.67 \\
\hline \multicolumn{12}{|l|}{$\begin{array}{l}\text { Comorbid } \\
\text { symptoms: }\end{array}$} \\
\hline CDI & 5.4 & 3.4 & 7.5 & 4.2 & 2.5 & 5.9 & 4.0 & 2.0 & 6.0 & 0.080 & - \\
\hline SNAP & $27.7^{\mathrm{a}}$ & 23.3 & 32.1 & $26.0^{\mathrm{a}}$ & 19.0 & 32.9 & $21.7^{\mathrm{b}}$ & 15.7 & 27.7 & 0.003 & 0.59 \\
\hline \multicolumn{12}{|l|}{ Quality of Life: } \\
\hline YQOL & 82.1 & 74.9 & 89.4 & 83.5 & 77.7 & 89.3 & 83.5 & 77.7 & 89.3 & 0.602 & - \\
\hline
\end{tabular}

Notes: Different letters $\left({ }^{\mathrm{a}, \mathrm{b}, \mathrm{c}}\right)$ indicate statistical significant results at 0.05 level of significant in sequential Bonferroni multiple comparison adjustment.

*Effect size (Cohen's formula) between baseline and the 14 week assessment.

Abbreviations: EEM, Estimated Marginal Means; CI95\%, 95\% Confidence Interval; GEE, Generalized Estimating Equations; PARS, Pediatric Anxiety Rating Scale; CGI, Clinical Global Impressions; CGAS, Children's Global Assessment Scale; SCARED-C, Screen for Child Anxiety Related Disorders - Child Version; SCARED-P, Screen for Child Anxiety Related Disorders - Parent Version; CDI, Childhood Depression Inventory; YQOL, Youth Quality of Life Instrument.

had ADHD comorbidity (Kendall, Brady and Verduin, 2001). Furthermore, improvement of anxiety symptoms may have contributed to the positive effects on externalizing symptoms (Beidas, Benjamin, Puleo, Edmunds and Kendall, 2010).

In terms of intervention delivery our experience suggests that the presence of a co-therapist in all sessions was essential. In terms of time, no session exceeded 90 minutes, with group size being limited to six due to individual problems of participants (shyness, anxiety). According to family report, the two sessions designed for family members were very important in understanding the disorder, learning how to deal with the child's anxiety and cooperating with the child's homework.

Our study has limitations that should be taken into consideration. The lack of a control group, the absence of a follow-up and the small sample size limit the generalization of our findings. Similarly, only $50 \%$ of those who were eligible actually completed the treatment. In spite of this, the effect size of interventions greatly encourages further research. The youths suffered from different kinds of anxiety disorders and, as the sample was too small, it remains unclear whether or not a specific anxiety disorder demonstrated a better response to group CBT. On the other hand, this was a sample directly selected from the community and not a treatment-seeking one. Given the high prevalence and substantial morbidity associated with anxiety disorders in childhood and adolescence, dissemination of CBT from research to clinical practice should be a priority (Beidas et al., 2010; Kendall et al., 2011; March, 
2011; McLean and Foa, 2011). However, an important barrier is the lack of CBT practitioners adequately trained in empirically supported treatments (Kendall et al., 2011). The use of a group CBT protocol aided the ability of clinicians to provide an effective manual driven treatment. Further, randomized controlled trials are needed to confirm our findings with an appropriate follow-up to evaluate whether gains are maintained over time.

\section{Acknowledgements}

Financing was project linked from Universal Edict - CNPq 2007 (Number: 483032/2007-7), FIPE-HCPA and CAPES-Prof.

\section{References}

Beesdo, K., Knappe, S. and Pine, D. S. (2009). Anxiety and anxiety disorders in children and adolescents: developmental issues and implications for DSM-V. Psychiatric Clinics of North America, 32, 483-524.

Beidas, R. S., Benjamin, C. L., Puleo, C. M., Edmunds, J. M. and Kendall, P. C. (2010). Flexible applications of the Coping Cat Program for anxious youth. Cognitive and Behavioral Practice, 17, $142-153$.

Birmaher, B., Khetarpal, S., Brent, D., Cully, M., Balach, L., Kaufman, J., et al. (1997). The Screen for Child Anxiety Related Emotional Disorders (SCARED): scale construction and psychometric characteristics. Journal of the American Academy of Child and Adolescent Psychiatry, 36, 545-553.

Braga, D. T., Manfro, G. G., Niederauer, K. and Cordioli, A. V. (2010). Full remission and relapse of obsessive-compulsive symptoms after cognitive-behavioral group therapy: a two-year follow-up. Revista Brasileira de Psiquiatria, 32, 164-168.

Connolly, S. D., Bernstein, G. A. and Issues, W. G. O. Q. (2007). Practice parameter for the assessment and treatment of children and adolescents with anxiety disorders. Journal of the American Academy of Child and Adolescent Psychiatry, 46, 267-283.

Cordioli, A. V., Heldt, E., Braga, D. B., Margis, R., Sousa, M. B., Tonello, J. F., et al. (2003). Cognitive-behavioral group therapy in obsessive-compulsive disorder: a randomized clinical trial. Psychotherapy and Psychosomatics, 72, 211-216.

Costello, E. J., Egger, H. L. and Angold, A. (2005). The developmental epidemiology of anxiety disorders: phenomenology, prevalence, and comorbidity. Child and Adolescent Psychiatric Clinics of North America, 14, 631-648.

Edwards, T. C., Huebner, C. E., Connell, F. A. and Patrick, D. L. (2002). Adolescent quality of life, Part I: conceptual and measurement model. Journal of Adolescence, 25, 275-286.

Golfeto, J. H. and D'Oliveira, A. B. (2004). Prevalence study of depressive symptomatology and the risk of suicide in school children in Ribeirão Preto evaluated by the Inventory of Child Depression (CDI)]. Revista Brasileira de Psiquiatria, 26, 68-69.

Guy, W. (1976). Clinical Global Impression (CGI). In ECDEU Assessment Manual for Psychopharmacology. US Department of Health and Human Services, Public Health Service, Alcohol Drug Abuse and Mental Health Administration, NIMH Psychopharmacology Research branch. Rockville, MD: National Institute of Mental Health, 218-222.

Heldt, E., Kipper, L., Blaya, C., Salum, G. A., Hirakata, V. N., Otto, M. W., et al. (2011). Predictors of relapse in the second follow-up year post cognitive-behavior therapy for panic disorder. Revista Brasileira de Psiquiatria, 33, 23-29.

Heldt, E., Manfro, G. G., Kipper, L., Blaya, C., Maltz, S., Isolan, L., et al. (2003). Treating medication-resistant panic disorder: predictors and outcome of cognitive-behavior therapy in a Brazilian public hospital. Psychotherapy and Psychosomatics, 72, 43-48. 
Isolan, L., Salum, G. A., Osowski, A. T., Amaro, E. and Manfro, G. G. (2011). Psychometric properties of the Screen for Child Anxiety Related Emotional Disorders (SCARED) in Brazilian children and adolescents. Journal of Anxiety Disorders, 25, 741-748.

James, A., Soler, A. and Weatherall, R. (2007). Cognitive Behavioural Therapy for Anxiety Disorders in Children and Adolescents (Cochrane Review). In The Cochrane Library, Issue 4. New York: Wiley and Sons.

Kaufman, J., Birmaher, B., Brent, D., Rao, U., Flynn, C., Moreci, P., et al. (1997). Schedule for Affective Disorders and Schizophrenia for School-Age Children-Present and Lifetime Version (KSADS-PL): initial reliability and validity data. Journal of the American Academy of Child and Adolescent Psychiatry, 36, 980-988.

Kendall, P. C., Brady, E. U. and Verduin, T. L. (2001). Comorbidity in childhood anxiety disorders and treatment outcome. Journal of the American Academy of Child and Adolescent Psychiatry, 40, 787-794.

Kendall, P. C., Flannery-Schroeder, E., Panichelli-Mindel, S. M., Southam-Gerow, M., Henin, A. and Warman, M. (1997). Therapy for youths with anxiety disorders: a second randomized clinical trial. Journal of Consulting and Clinical Psychology, 65, 366-380.

Kendall, P. C. and Hedtke, K. A. (2006a). Cognitive-Behavioral Therapy for Anxious Children: therapist manual for group treatment. Coping Cat (3rd ed.). Pennsylvania: Workbook Publishing.

Kendall, P. C. and Hedtke, K. A. (2006b). The Coping Cat Workbook (2nd ed.). Pennsylvania: Workbook Publishing.

Kendall, P. C., Khanna, M. S., Edson, A., Cummings, C. and Harris, M. S. (2011). Computers and psychosocial treatment for child anxiety: recent advances and ongoing efforts. Depression and Anxiety, 28, 58-66.

Kendall, P. C., Safford, S., Flannery-Schroeder, E. and Webb, A. (2004). Child anxiety treatment: outcomes in adolescence and impact on substance use and depression at 7.4-year follow-up. Journal of Consulting and Clinical Psychology, 72, 276-287.

Kessler, R. C., Berglund, P., Demler, O., Jin, R., Merikangas, K. R. and Walters, E. E. (2005). Lifetime prevalence and age-of-onset distributions of DSM-IV disorders in the National Comorbidity Survey Replication. Archives of General Psychiatry, 62, 593-602.

Klein, R. G. (2009). Anxiety disorders. Journal of Child Psychology and Psychiatry, 50, 153162.

March, J. S. (2011). Looking to the future of research in pediatric anxiety disorders. Depression and Anxiety, 28, 88-98.

McLean, C. P. and Foa, E. B. (2011). Prolonged exposure therapy for post-traumatic stress disorder: a review of evidence and dissemination. Expert Review of Neurotherapeutics, 11, 1151-1163.

Merikangas, K. R., He, J. P., Burstein, M., Swanson, S. A., Avenevoli, S., Cui, L., et al. (2010). Lifetime prevalence of mental disorders in US adolescents: results from the National Comorbidity Survey Replication-Adolescent Supplement (NCS-A). Journal of the American Academy of Child and Adolescent Psychiatry, 49, 980-989.

Patrick, D. L., Edwards, T. C. and Topolski, T. D. (2002). Adolescent quality of life, part II: initial validation of a new instrument. Journal of Adolescence, 25, 287-300.

The Research Units on Pediatric Psychopharmacology Anxiety Study Group (2002). The Pediatric Anxiety Rating Scale (PARS): development and psychometric properties. (2002). Journal of the American Academy of Child and Adolescent Psychiatry, 41, 1061-1069.

Roza, S. J., Hofstra, M. B., Van Der Ende, J. and Verhulst, F. C. (2003). Stable prediction of mood and anxiety disorders based on behavioral and emotional problems in childhood: a 14-year follow-up during childhood, adolescence, and young adulthood. American Journal of Psychiatry, 160, 21162121.

Rynn, M., Puliafico, A., Heleniak, C., Rikhi, P., Ghalib, K. and Vidair, H. (2011). Advances in pharmacotherapy for pediatric anxiety disorders. Depression and Anxiety, 28, 76-87. 
Salum, G. A., Isolan, L. R., Bosa, V. L., Tocchetto, A. G., Teche, S. P., Schuch, I., et al. (2011). The multidimensional evaluation and treatment of anxiety in children and adolescents: rationale, design, methods and preliminary findings. Revista Brasileira de Psiquiatria, 33, 181-195.

Serra-Pinheiro, M. A., Mattos, P. and Angélica, R. M. (2008). Inattention, hyperactivity, and oppositional-defiant symptoms in Brazilian adolescents: gender prevalence and agreement between teachers and parents in a non-English speaking population. Journal of Attention Disorders, 12, 135140 .

Shaffer, D., Gould, M. S., Brasic, J., Ambrosini, P., Fisher, P., Bird, H., et al. (1983). A children's global assessment scale (CGAS). Archives of General Psychiatry, 40, 1228-1231.

Walkup, J. T., Albano, A. M., Piacentini, J., Birmaher, B., Compton, S. N., Sherrill, J. T., et al. (2008). Cognitive behavioral therapy, sertraline, or a combination in childhood anxiety. The New England Journal of Medicine, 359, 2753-2766. 